Pacific Journal of Mathematics

MBEDDING PUNCTURED LENS SPACES AND CONNECTED
SUMS 


\title{
IMBEDDING PUNCTURED LENS SPACES AND CONNECTED SUMS
}

\author{
DANIEL RUBERMAN
}

\begin{abstract}
We investigate codimension-one imbeddings of punctured lens spaces and connected sums of lens spaces. For $\left|\pi_{1}(L)\right|$ a prime power we show that $L-B^{2 k-1}$ imbeds in $S^{2 k}$ if and only if $L$ is of a certain special form. If $L \# L^{\prime}$ imbeds in $S^{2 k}$, then $L \simeq L^{\prime}$ and $L$ is homology cobordant to $L^{\prime}$. For $\left|\pi_{1}(L)\right|$ a prime power, this implies (via Smith-theory) that $L \cong L^{\prime}$.
\end{abstract}

Introduction. When does a manifold imbed with codimension one in Euclidean space? We investigate this question for lens spaces and manifolds made from lens spaces. It is not hard to show that $L^{2 k-1}$ never imbeds in $S^{2 k}$ - see the remark after Theorem 6. However, the following two questions are more subtle and are the focus of this paper.

Problem A. Which punctured lens spaces $L_{0}\left(=L-B^{2 k-1}\right)$ imbed in $S^{2 k} ?$

Problem B. If $L_{0}$ and $L_{0}^{\prime}$ both imbed in $S^{2 k}$, does $L \# L^{\prime}$ ?

Problem A is settled in the classical three-dimensional case: by Zeeman's twist-spinning construction and the work of Epstein [5] a punctured lens space $L_{0}(m ; q, 1)$ imbeds in $S^{4}$ if and only if $m$ is odd. Problem B was treated in the classical case by Livingston and Gilmer [8] so the discussion below is limited to $k>2$.

The first obstruction to codimension-one imbedding that one might look for is the tangent bundle. For if $L_{0}$ imbeds in $S^{2 k}, \tau(L)$ is stably trivial. Ewing et al. [6] examine the question of when a lens space with $\left|\pi_{1}(L)\right|$ a prime is stably parallelizable and give one simple class of lens spaces that are (Proposition 2.1 of [6]). These all actually imbed punctured; in fact a considerably larger class (which we refer to as the class $\mathscr{G}$ ) of lens spaces all imbed - see Theorem 5. For $\left|\pi_{1}(L)\right|=$ a prime power, we show in Theorem 9 that $L_{0}$ imbeds in $S^{2 k}$ if and only if $L \in \mathcal{9}$, and conjecture that this holds in general, i.e.:

Conjecture A. $L_{0}$ imbeds in $S^{2 k}$ if and only if $L \in \mathcal{G}$. 
As for problem B, note the following elementary fact. If $L_{0}$ imbeds in $S^{2 k}$, then it has trivial normal bundle so $L \#-L=\partial\left(L_{0} \times I\right)$ imbeds in $S^{2 k}$. We will see that if $L \#-L^{\prime}$ imbeds in $S^{2 k}$, then $L \simeq L^{\prime}$ (preserving orientation). The most optimistic guess as to the answer to problem $B$ is:

Conjecture $B$. If $L \#-L^{\prime}$ imbeds in $S^{2 k}$ then $L \cong L^{\prime}$.

Again this conjecture holds if $\left|\pi_{1}\right|$ is a prime power. When it is not a prime power, life becomes more interesting. We do not show that $L \cong L^{\prime}$ but find a relationship between various $\alpha$-invariants of the lens spaces. This relationship is most conveniently stated in terms of the invariants defined by Casson and Gordon [2] to study knot concordance. We will use the invariants $\sigma(M, \psi)$ as defined in $\S 1$ of [7] and refer the reader to that paper for notation and definitions.

The material in this paper is part of my thesis. I would like to thank my advisor, Rob Kirby, for his help and direction. Conversations with Pat Gilmer and David Schorow were very helpful; I would like to thank them for their encouragement.

Lens spaces. Let $m$ be an integer and $q_{1} \cdots q_{k}$ integers with $\left(m, q_{i}\right)=$ 1. The cyclic group $\mathbf{Z}_{m}$ acts on $\mathbf{C}^{k}$ : if $T \in \mathbf{Z}_{m}$ is the generator and $\omega=e^{2 \pi i / m}$, then $T\left(z_{1} \cdots z_{k}\right)=\left(\omega^{q_{1}} z_{1}, \ldots, \omega^{q_{k}} z_{k}\right)$. This restricts to a free action on $S^{2 k-1}$; the quotient is denoted $L\left(m ; q_{1} \cdots q_{k}\right)$. There is a preferred orientation of $L$ (coming from $S^{2 k-1}$ ) which we fix, and a preferred generator, denoted $g$, of $\pi_{1}(L) \cong \mathbf{Z}_{m}$ corresponding to the covering translation $T$. For each $q_{j}$, choose an integer $r_{j}$ with $r_{j} q_{j} \equiv 1$ $(\bmod m)$.

As is well known the classifying space $B \mathbf{Z}_{m} \simeq K\left(\mathbf{Z}_{m}, 1\right)$ can be considered as an infinite lens space $L(m ; 1,1,1, \ldots)$ and we will always think of it this way. As such, it too has a canonical generator $\bar{g}$ for $\pi_{1}$. The following is known [4] and summarizes the homology and cohomology structure of the lens spaces. For $p$ an integer, let

$$
\nu: H^{j}\left(, \mathbf{Z}_{p}\right) \rightarrow H^{j+1}\left(, \mathbf{Z}_{p}\right)
$$

be the Bockstein coboundary corresponding to the coefficient sequence $0 \rightarrow \mathbf{Z}_{p} \rightarrow \mathbf{Z}_{p^{2}} \rightarrow \mathbf{Z}_{p} \rightarrow 0$.

Proposition 1. Let $L=L\left(m ; q_{1} \cdots q_{k}\right)$. Then

$$
H_{j}(L)= \begin{cases}\mathbf{Z} & j=0,2 k-1, \\ \mathbf{Z}_{m} & j \text { odd }<2 k-1, \\ 0 & \text { jeven } .\end{cases}
$$


If $p$ is a prime dividing $m$, then $H^{j}\left(L ; \mathbf{Z}_{p}\right) \cong \mathbf{Z}_{p}($ all $j \leq 2 k-1)$. Further, any generator $\alpha$ of $H^{1}\left(L ; \mathbf{Z}_{p}\right)$ has the property that $(\nu \alpha)^{j}$ generates $H^{2 j}\left(L ; \mathbf{Z}_{p}\right)$, and $\alpha \cdot(\nu \alpha)^{j}$ generates $H^{2 j+1}\left(L ; \mathbf{Z}_{p}\right)$. The corresponding statements are true for $B \mathbf{Z}_{m}$ if $k=\infty$.

Cohen [3] constructs an explicit cell decomposition for any $L(m$; $q_{1} \cdots q_{2}$ ) which works for $B \mathbf{Z}_{m}$ as well. Call the generator of $H_{2 j-1}(L)$ arising from this cell-decomposition $e_{2 j-1}$; likewise $\bar{e}_{2 j-1}$ is the generator of $H_{2 j-1}\left(B Z_{m}\right)$. Note that with these conventions $g=q_{1} e_{1}$ and $\bar{g}=\bar{e}_{1}$ where $g$ and $\bar{g}$ are the generators mentioned above. Since $\operatorname{Hom}\left(H_{1}(L)\right.$; $\left.\mathbf{Z}_{d}\right)=\left[L, B \mathbf{Z}_{d}\right], \phi_{*}: H_{*}(L) \rightarrow H_{*}\left(B \mathbf{Z}_{d}\right)$ depends only on $\phi: H_{1}(L) \rightarrow$ $H_{1}\left(B \mathbf{Z}_{d}\right)=\mathbf{Z}_{d}$. Cohen ([3] \$29) constructs explicit maps realising any homomorphism $\phi: H_{1}(L) \rightarrow \mathbf{Z}_{m}$; a similar construction works for any $\alpha$ dividing $m$. It is an easy matter to calculate $\phi_{*}: H_{*}(L) \rightarrow H_{*}\left(B \mathbf{Z}_{d}\right)$ using these representatives. (Compare [3] theorem 29.4.)

Proposition 2. Suppose the character $\phi: H_{*}(L) \rightarrow \mathbf{Z}_{d}(m=d \cdot n)$ is given by $\phi(g)=r$. Then $\phi_{*}\left(e_{2 j-1}\right)=(n r)^{j} r_{1} \cdots r_{j} \bar{e}_{2 j-1}$.

Similarly, one can calculate the effect on homology of a map $f$ : $L \rightarrow L$.

PROPOSITION 3. If $f: L \rightarrow L$ is a map with $f_{*}(g)=r g$, then $f_{*}\left(e_{2 j-1}\right)$ $=r^{j} e_{2 j-1}$, and the degree of $f$ is $r^{k}(\bmod m)$.

Some of the examples presented below involve $(4 k-1)$ dimensional lens spaces $L\left(m ; q_{1}, \ldots, q_{2 k}\right)$; for these we need to compute the linking form $\lambda: H_{2 k-1}(L) \times H_{2 k-1}(L) \rightarrow \mathbf{Q} / \mathbf{Z}$. This computation dates back to deRham [10]. In terms of the generator $e$, the answer is given by:

PROPOSITION 4. $\lambda(e, e)=q_{1} \cdots q_{k} r_{k+1} \cdots r_{2 k} / m\left(q_{j} r_{j} \equiv 1(m)\right)$. It is convenient to set $f=q_{k+1} \cdots q_{2 k} e$; then $\lambda(f, f)=q_{1} \cdots q_{2 k} / m=q / m$ $\left(q=q_{1} \cdots q_{2 k}\right)$, which is what one might expect by analogy with classical lens spaces.

Imbedding punctured lens spaces. We now present the class 9 of lens spaces which imbed punctured with codimension one in the sphere.

Let $\left(*_{k}\right)$ be the condition: $c^{k} \equiv 1(m)$ but $c^{j}-1$ is a unit $\bmod m$ for $j<k$. 
THEOREM 5. (a) Suppose $c$ satisfies the condition $\left(*_{k}\right)$. Then $L\left(m ; 1, c, \ldots, c^{k-1}\right)$ imbeds punctured in $S^{2 k}(k \geq 2)$. (b) If $b$ is any unit $\bmod m$, and $c$ satisfies $\left(*_{2 k}\right)$ then $L_{0}\left(m ; 1, c, \ldots, c^{k-1}, b, b c, \ldots, b c^{k-1}\right)$ imbeds in $S^{4 k}(k>1)$.

Proof. (a) Cohen ([3], §31) constructs a diffeomorphism $f: L \rightarrow L$ with $f_{*}\left(g_{1}\right)=c g$. (The point is that $c \cdot\left\{1, c, \ldots, c^{k-1}\right\} \equiv\left\{1, c, \ldots, c^{k-1}\right\}$.) Consider the mapping torus $S^{1} \times{ }_{f} L_{0}$; by construction $f$ has degree 1 we can assume it to be the identity on the top cell, so $\partial\left(S^{1} \times_{f} L_{0}\right)=S^{1} \times$ $S^{2 k-2}$.

Claim. $S^{1} \times_{f} L_{0} \cup_{\partial} D^{2} \times S^{2 k-2} \simeq S^{2 k}$.

Proof of Claim. $\pi_{1}\left(S^{1} \times{ }_{f} L_{0}\right)$ is a standard HNN construction and is given by $\left\langle t, g \mid t g t^{-1}=g^{c}, g^{m}=1\right\rangle$. Adding on the $D^{2}$ kills $t$, so $\pi_{1}$ becomes $\left\langle g \mid g^{c-1}=1, g^{m}=1\right\rangle$. Since $c-1$ is a unit $\bmod m, \pi_{1}$ is trivial. The Wang sequence for $S^{1} \times_{f} L_{0}$ is

$$
H_{j+1}\left(S^{1} \times_{f} L_{0}\right) \rightarrow H_{j}\left(L_{0}\right)^{f_{*}^{-1}} \rightarrow H_{j}\left(L_{0}\right) \rightarrow H_{j}\left(S^{1} \times_{f} L_{0}\right) \rightarrow \cdots .
$$

but $f_{*}: H_{j} L_{0} \rightarrow H_{j} L_{0}$ is multiplication by $c^{j}$ so the assumption on $c$ says that $f_{*}-1$ is an isomorphism. This together with the computation that $\pi_{1}$ is trivial proves the claim.

Now $L_{0}$ is imbedded in a smooth homotopy sphere which is therefore homeomorphic to $S^{2 k}$. Connect summing with another (possibly fake) homotopy sphere away from a copy of $L_{0}$ gives an imbedding of $L_{0}$ in the real $S^{2 k}$.

The proof of (b) is the same once one notes that $c$ satisfies $\left(*_{2 k}\right) \Rightarrow c^{k}$ $\equiv-1(\bmod m)$. For $\left(c^{k}+1\right)\left(c^{k}-1\right)=c^{2 k}-1 \equiv O(m)$ and $c^{k}-1$ is a unit by assumption. Thus

$$
\begin{aligned}
c \cdot\left\{1, c, \ldots, c^{k-1}, b, b c, \ldots, b c^{k-1}\right\} & \\
& =\left\{-1,-c, \ldots,-c^{k-1},-b,-b c, \ldots,-b c^{k-1}\right\} .
\end{aligned}
$$

Since there are an even number of minus signs, there is indeed an orientation preserving diffeomorphism of $L$ inducing $g \rightarrow c g$ on $\pi_{1}$.

In either case (a) or (b), we say that $L \in \mathscr{G}$. As we remarked earlier, the theorem is true for classical lens spaces as well. In fact, the imbedding provided by part (b) (choose $c=-1$ ) which a priori lies in a homotopy 4-sphere is the same as the imbedding in the real sphere given by twist-spinning a rational knot. Note that -1 satisfies $\left(*_{4}\right)$ if and only if $m$ is odd. 
There are many stably parallelizable lens spaces other than those in $\mathscr{Y}$; see [6]. It is tempting to believe that none of these others imbed, i.e. that conjecture $A$ holds. This conjecture is true whenever $m$ is a prime power as will be shown below in Theorem 9 .

Obstructions to imbedding. The same theorem underlies our attack on both problems A and B. Let $M=L\left(m ; q_{1}, \ldots, q_{k}\right) \#-L^{\prime}\left(m^{\prime} ; q_{1}^{\prime}, \ldots, q_{k}^{\prime}\right)$. For $p$ a prime write $H_{j}(X)_{(p)}$ for those elements of $H_{j}(X)$ annihilated by a power of $p$ (i.e. the $p$-torsion of $H_{j}(X)$ ).

TheOREM 6. Suppose $M$ imbeds in $S^{2 k}$ with $S^{2 k}-M=W \cup W^{\prime}$. Then $W\left(\right.$ and $\left.W^{\prime}\right)$ is a homology cobordism between $L_{0}$ and $L_{0}^{\prime}$.

Proof. We establish a series of assertions. Note first that $H_{*}(W)$ and $H_{*}\left(W^{\prime}\right)$ are both torsion as are $H^{*}(W)$ and $H^{*}\left(W^{\prime}\right)$.

Claim 1. For all primes $p$ dividing $m$,

$$
H^{1}\left(W ; \mathbf{Z}_{p}\right) \neq 0 \text { and } H^{1}\left(W^{\prime} ; \mathbf{Z}_{p}\right) \neq 0 .
$$

Proof. If, say, $H^{1}\left(W^{\prime} ; \mathbf{Z}_{p}\right)=0$, then $H_{1}\left(W^{\prime}\right)_{(p)}=0$. But then $i_{*}$ : $H_{1}(M)_{(p)} \stackrel{\cong}{\rightrightarrows} H_{1}(W)_{(p)}$ by the Mayer-Vietoris sequence. So any character on $M$ of order a power of $p$ is null-bordant, since such a character extends over $W$. Write $m=p^{n} s$ where $(s, p)=1$, and consider the character on $M$ given by $\psi(g)=1, \psi\left(g^{\prime}\right)=0$ where $g, g^{\prime}$ generate $H_{1}\left(L_{0}\right)$ and $H_{1}\left(L_{0}^{\prime}\right)$.

Now $(M, \psi)=(L, \psi)-\left(L^{\prime}, \psi^{\prime}\right)$ in the bordism group $\Omega_{2 k-1}\left(\mathbf{Z}_{p^{n}}\right)$. By assumption $L^{\prime}-$ int $B^{2 k-1}$ is imbedded in $S^{2 k}$; its boundary is a slice knot, so $L^{\prime}$ imbeds in $B^{2 k+1}$ and hence in $S^{2 k+1}$. By a standard transversality argument, $L^{\prime}$ bounds some oriented $2 k$-manifold $V$. Since the map $\psi^{\prime}$ is trivial it extends over $V$ and so $\left(L^{\prime}, \psi^{\prime}\right)=0$ in $\Omega_{2 k-1}\left(\mathbf{Z}_{p^{n}}\right)$. It follows that $(L, \psi)=0$ as well and hence that $\psi_{*}\left(e_{2 k-1}\right)=0$ in $H_{2 k-1}\left(B Z_{p^{n}}\right)$. But by Proposition 2, $\psi_{*}\left(e_{2 k-1}\right)=s^{k} r_{1} \cdots r_{k} \bar{e}_{2 k-1}$ which is non-trivial since $(s, p)=\left(r_{i}, p\right)=1$.

Claim 2. If $p$ divides $m$ or $m^{\prime}$, then $H^{1}\left(W ; \mathbf{Z}_{p}\right)=\mathbf{Z}_{p}$, and

$$
i^{*}: H^{\mathrm{I}}\left(W ; \mathbf{Z}_{p}\right) \rightarrow H^{1}\left(M ; \mathbf{Z}_{p}\right)
$$

is an injection. The same is true for $W^{\prime}$. 
Proof. Suppose $p$ divides $m . H_{1}(M)$ is a direct sum of two cyclic groups, so $H^{1}\left(M ; \mathbf{Z}_{p}\right)=\mathbf{Z}_{p}$ or $\mathbf{Z}_{p}+\mathbf{Z}_{p}$. The $\mathbf{Z}_{p}$ Mayer-Vietoris sequence reads:

$$
0 \rightarrow \begin{gathered}
H^{1}\left(W ; \mathbf{Z}_{p}\right) \\
+ \\
H^{1}\left(W^{\prime} ; \mathbf{Z}_{p}\right)
\end{gathered} \stackrel{i^{*}+i^{\prime *}}{\rightarrow} H^{1}\left(M ; \mathbf{Z}_{p}\right) \rightarrow 0
$$

so that $i^{*}$ and $i^{\prime *}$ are both injections. Since neither $H^{1}\left(W ; \mathbf{Z}_{p}\right)$ nor $H^{1}\left(W^{\prime} ; \mathbf{Z}_{p}\right)$ is zero, they must both be $\mathbf{Z}_{p}$.

Claim 3. At least one of the maps $H^{1}\left(W ; \mathbf{Z}_{p}\right) \rightarrow H^{1}\left(L_{0} ; \mathbf{Z}_{p}\right)$ or $\rightarrow H^{1}\left(L_{0}^{\prime} ; \mathbf{Z}_{p}\right)$ is an isomorphism, and likewise for $W^{\prime}$.

Proof. All of the groups in question are $\mathbf{Z}_{p}$, and a homomorphism $\mathbf{Z}_{p} \rightarrow \mathbf{Z}_{p}$ is either zero or an isomorphism. If both are zero then $i^{*}: H^{1}\left(W ; \mathbf{Z}_{p}\right) \rightarrow H^{1}\left(M ; \mathbf{Z}_{p}\right)$ is zero, contradicting claim 2 .

Claim 4. If $i^{*}: H^{1}\left(W ; \mathbf{Z}_{p}\right) \rightarrow H^{1}\left(L_{0} ; \mathbf{Z}_{p}\right)$ is an isomorphism, then $i^{*}$ : $H^{j}\left(W ; \mathbf{Z}_{p}\right) \rightarrow H^{j}\left(L_{0} ; \mathbf{Z}_{p}\right)$ is onto for all $j$.

Proof. Choose $a \in H^{1}\left(W ; \mathbf{Z}_{p}\right)$ such that $i^{*}(a)$ generates $H^{1}\left(L_{0} ; \mathbf{Z}_{p}\right)$. Then $i^{*}\left((\nu a)^{j}\right)$ generates $H^{2 j}\left(L_{0} ; \mathbf{Z}_{p}\right)$ and $i^{*}\left(a(\nu a)^{j}\right)$ generates $H^{2 j+1}\left(L_{0} ; \mathbf{Z}_{p}\right)$ by Proposition 1 and the naturality of the cup-product and Bockstein.

Claim 4 finishes the theorem, for it shows that $\left|H^{j}\left(W ; \mathbf{Z}_{p}\right)\right| \geq p$ and similarly that $\left|H^{j}\left(W^{\prime} ; \mathbf{Z}_{p}\right)\right| \geq p$. Now the Mayer-Vietoris sequence

$$
0 \rightarrow \underset{H^{j}\left(W^{\prime} ; \mathbf{Z}_{p}\right)}{+} \rightarrow H^{j}\left(M ; \mathbf{Z}_{p}\right)=\mathbf{Z}_{p}+\mathbf{Z}_{p} \rightarrow 0
$$

shows that $H^{j}\left(W ; \mathbf{Z}_{p}\right)=\mathbf{Z}_{p}$. Therefore $i^{*}: H^{j}\left(W ; \mathbf{Z}_{p}\right) \rightarrow H^{j}\left(L_{0} ; \mathbf{Z}_{p}\right)$ is an isomorphism for all $j$, and so $H^{*}\left(W, L_{0}: \mathbf{Z}_{p}\right)=0$, or equivalently, $H_{*}\left(W, L_{0}\right)_{(p)}=0$. By duality (see [9]) $H^{j}\left(W, L_{0}^{\prime}\right)_{(p)}=H_{2 k-j}\left(W, L_{0}\right)_{(p)}$ $=0$ and hence $H_{*}\left(W, L_{0}^{\prime}\right)_{(p)}=0$. Repeating this argument for each $p$ dividing $m$, and possibly interchanging the roles of $L_{0}$ and $L_{0}^{\prime}$ shows that for all primes, $H_{*}\left(W, L_{0}\right)_{(p)}=0$. Since $H_{*}\left(W, L_{0}\right)$ is torsion, $H_{*}\left(W, L_{0}\right)$ is zero. Hence $\left(W, L_{0}, L_{0}^{\prime}\right)$ is a homology bordism; the same argument shows that $\left(W^{\prime}, L_{0}, L_{0}^{\prime}\right)$ is also a homology bordism.

REMARK. One can "cap off” $W$ (or $\left.W^{\prime}\right)$ by adding a $(2 k-1)$-handle to $W$ along the separating $(2 k-2)$-sphere in $L \#-L^{\prime}$ to obtain homology bordisms between $L$ and $L^{\prime}$. This justifies the statement made in the 
introduction that $L$ never imbeds in $S^{2 k}$. For $L=L \# S^{2 k-1}$; if $L \subset S^{2 k}$, we have the absurdity that $L$ is homology bordant to $S^{2 k-1}$. This can also be shown directly using the method of Theorem 6 .

COROLlaRY 7. If $L \#-L^{\prime}$ imbeds in $S^{2 k}$ then $L \simeq L^{\prime}$ (preserving orientation).

Proof. We have a homology bordism $\left(V, L, L^{\prime}\right)$ by the preceding remark. By obstruction theory there is a retraction $r: V \rightarrow L^{\prime}$, set $f=r \mid L$. Then $f$ is orientation preserving and is an isomorphism on homology and hence is a homotopy equivalence.

LEMMA 8. $A \mathbf{Z}_{p^{r}}$-cover of a homology bordism $\left(V, N, N^{\prime}\right)$ is a rational homology bordism.

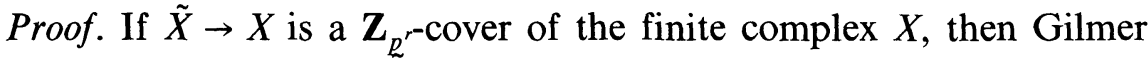
proves $\left(1.3\right.$ of [7]) that $\beta_{j}(\tilde{X})-\beta_{j}(X) \leq\left(p^{r}-1\right) \beta_{j}\left(X ; \mathbf{Z}_{p}\right)$ using Smith theory. The entire discussion goes through for pairs $(X, Y)$ such that the induced cover of the subcomplex $Y$ is connected, and one obtains $\beta_{j}(\tilde{X}, \tilde{Y})-\beta_{j}(X, Y) \leq\left(p^{r}-1\right) \beta_{j}\left(X, Y ; \mathbf{Z}_{p}\right)$. In our case, $\beta_{j}(V, N)=$ $\beta_{j}\left(V, N ; \mathbf{Z}_{p}\right)=0$, so we obtain $\beta_{j}(\tilde{V}, \tilde{N})=0$. In other words, $\left(\tilde{V}, \tilde{N}, \tilde{N^{\prime}}\right)$ is a $Q$-homology bordism.

Lemma 8 implies that for characters of prime-power order extending over a homology bordism the Casson-Gordon invariants of the ends are equal. Equivalently, the corresponding $\alpha$-invariants of the covers are equal. This principle is the key to our best result on problem A.

THEOREM 9. Suppose $L_{0}\left(p^{r} ; q_{1} \cdots q_{k}\right)$ imbeds in $S^{2 k}$.

(a) If $k$ is odd then for some c satisfying $\left(*_{k}\right) L \cong L\left(p^{r} ; 1, c, \ldots, c^{k-1}\right)$.

(b) If $k$ is even, say $k=2 n$, then for some unit $b$ and some $c$ satisfying $\left(*_{k}\right), L \cong L\left(p^{r} ; 1, c, \ldots, c^{n-1}, b, b c, \ldots, b c^{n-1}\right)$.

Proof. It is not hard to see that the theorem will follow in both cases if we find $c$ such that $c \cdot\left\{q_{1} \cdots q_{k}\right\}=\left\{ \pm q_{1} \cdots \pm q_{k}\right\}$, and $\left(c^{j}-1, p\right)=$ $1(j<k)$.

Set $W=S^{2 k}-L_{0} \times(0,1)=$ one component of $S^{2 k}-(L \#-L)$. By Theorem $6 W$ is a homology bordism between $L_{0}$ and $L_{0}$; "cap it off" as in Corollary 7 to obtain a bordism $V$ between $L$ and $L$. As noted in Corollary 7 , there is a homotopy equivalence $f: L \rightarrow L$ obtained by retracting $V$ onto one end. Determine $c$ by $f_{*}(g)=c \cdot g$. 
Now $\partial L_{0}$ is a knot in $S^{2 k}$ and its exterior is a homology circle which is made up of $W$ and $L_{0} \times I$.

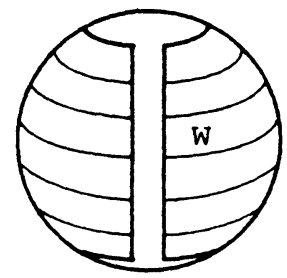

The Mayer-Vietoris sequence for the complement as the union of these two pieces reduces to

$$
0 \rightarrow H_{j}\left(L_{0}\right) \stackrel{f_{*}^{-1}}{\rightarrow} H_{j}\left(L_{0}\right) \rightarrow 0 \text { for } j>0,
$$

exactly as in the Wang sequence for a fibered knot. Since $f_{*}$ is multiplication by $c^{j}$ in dimensions $2 j-1$, we obtain $\left(c^{j}-1, p\right)=1$.

By Lemma 8, the $\mathbf{Z}_{p^{r}}$-cover of $V$ is a rational homology bordism from $S^{2 k-1}$ to itself. Hence $\forall t \in \mathbf{Z}_{p^{r}}$ the $t$-signatures of $S^{2 k}$, given by the restriction of the action of $\mathbf{Z}_{p^{r}}$ on $\tilde{V}$ to either end must agree. In other words $\operatorname{sign}\left(t, S^{2 k-1}\right)=\operatorname{sign}\left(t^{c}, S^{2 k-1}\right) \forall t \in \mathbf{Z}_{p^{r}}$. The argument of Atiyah and Bott ([1] Theorem 7.27) now shows that $\left\{c q_{1} \cdots c q_{k}\right\}=\left\{ \pm q_{1}\right.$ $\left.\cdots \pm q_{k}\right\}$.

REMARKs. (1) The same argument will apply even if $m$ is not a prime power if $L_{0}$ is assumed to be the fiber of a fibered knot. For then $V$ is actually an $h$-cobordism and one obtains the equality between the $\alpha$-invariants without the Smith-Theory argument.

(2) An example of a stably-parallelizable lens space that does not imbed punctured is $L(p ; 1, \ldots, 1)$ ( $p$ l's) for $p$ prime.

If $k$ is even then $L_{0}\left(m ; q_{1} \cdots q_{k}\right) \subset S^{2 k}$ implies that the linking form on $L \#-L$ is hyperbolic (see Theorem 10) and so by the argument in [8], p. $8 \mathrm{~m}$ must be odd. A nice corollary of the method in Theorem 9 is that $m$ must be odd, even when $k$ is odd. For in the proof of Theorem 9 we noted that there is an integer $c$ with $\left(c^{j}-1, m\right)=1$ for $0<j \leq k-1$. Suppose $m$ is even; then since $f$ is invertible $c$ must be odd. But then $c-1$ is even so $(c-1, m) \neq 1$.

Imbedding connected sums. Theorem 6 and Lemma 8 combine in a similar way to solve Problem B in the case that $m$ is a prime power. For if $L \#-L^{\prime}$ imbeds in $S^{2 k}$, then we have a homology bordism between $L$ and $L^{\prime}$ and so all the $\alpha$-invariants associated to prime-power covers are the 
same. If $m=p^{r}$, these are all the $\alpha$-invariants, and so the Atiyah-Bott result shows that $L \cong L^{\prime}$. If $m$ is not a prime-power, then we cannot conclude that $L \cong L^{\prime}$ because the prime-power $\alpha$-invariants alone do not determine $L$ - compare [8].

In general there is an ambiguity in exactly which $\alpha$-invariants are equal. However for $4 k-1$ dimensional lens spaces there is an interaction with the linking form that narrows the possibilities considerably. As usual we write $M=L\left(m ; q_{1}, \ldots, q_{2 k}\right) \#-L\left(m ; q_{1}^{\prime}, \ldots, q_{2 k}^{\prime}\right)$. It is more convenient to state the result in terms of the Casson-Gordon invariants of $L$ rather than the $t$-signatures of $\tilde{L}$; it can be translated if one desires. If $d \mid m$ let $\psi$ be the homomorphism from $H_{1}(L)$ to $\mathbf{Z}_{d}$ that gives 1 on $g$ and define $\psi^{\prime}$ similarly on $H_{1}\left(L^{\prime}\right)$.

THeOREM 10. Suppose $M$ imbeds in $S^{4 k}$. Let $p$ be a prime such that $p^{r} \mid m, p^{r+1} \nmid m$. Then for all $s$, there exist numbers $\alpha$ and $t$ such that:

(i) $\alpha^{2} q \equiv q^{\prime}\left(\bmod p^{r}\right)$

(ii) $\alpha s^{k} q_{1}^{\prime} \cdots q_{k}^{\prime} q_{k+1} \cdots q_{2 k}+t^{k} q_{1} \cdots q_{k} q_{k+1}^{\prime} \cdots q_{2 k}^{\prime} \equiv 0\left(\bmod p^{r}\right)$

(iii) $\sigma(L, s \psi)=\sigma\left(L^{\prime}, t \psi^{\prime}\right)$.

Proof. Let $W$ and $W^{\prime}$ be the components of $S^{4 k}-M$, and set $G=\operatorname{ker} i_{*}: H_{2 k-1}(M) \rightarrow H_{2 k-1}(W)$ and define $G^{\prime}$ likewise. Since $W$ and $W^{\prime}$ are homology bordisms, $G \cong G^{\prime} \cong \mathbf{Z}_{m}$, and we can write $H_{2 k-1}(M)=$ $G+G^{\prime}$. Further, the linking form $\lambda$ must vanish on $G$ and $G^{\prime}$, by a standard argument. With respect to the basis $f, f^{\prime}$ for $H_{2 k-1}(M), \lambda$ is given by the matrix

$$
\left(\begin{array}{cc}
q / p & 0 \\
0 & -q^{\prime} / p
\end{array}\right)
$$

Let $x$ and $y$ generate the summands $G$ and $G^{\prime}$, and write $x=\alpha f+\beta f^{\prime}$ and $y=\gamma f+\delta f^{\prime}$ with $\alpha \delta-\beta \gamma=$ unit $(\bmod m)$.

We have $0=\lambda(x, x)=(1 / m)\left(q \alpha^{2}-q^{\prime} \beta^{2}\right)(\bmod \mathbf{Z})$ and $0=$ $(1 / m)\left(q \gamma^{2}-q^{\prime} \delta^{2}\right)$, or $q \alpha^{2} \equiv q^{\prime} \beta^{2}(\bmod m)$ and $q \gamma^{2} \equiv q^{\prime} \delta^{2}(\bmod m)$. Since $\alpha \delta-\beta \gamma$ is a unit $\bmod m, \alpha, \beta, \gamma$, and $\delta$ can be assumed to be units as well. For $q \alpha^{2}=q^{\prime} \beta^{2}+j m$ so if, say, $\beta$ and $m$ have a common factor, $\alpha$ and $m$ have the same common factor. But this would contradict $(\alpha \delta-$ $\beta \gamma, m)=1$. So we may as well assume $\beta=\delta=1$; then $x=\alpha f+f^{\prime}$ and $y=\gamma f+f^{\prime}$ where $\alpha$ and $\gamma$ are roots of $q z^{2}-q^{\prime} \equiv 0(\bmod m)$.

Because $W$ is a homology cobordism, for each $s$ there is a $t$ such that the homomorphism $\Psi: H_{1}(M) \rightarrow \mathbf{Z}_{p^{r}}$ given by $\Psi=s \psi+t \psi^{\prime}$ extends over $H_{1}(W)$. For such a homomorphism, the induced map $\Psi_{*}: H_{*}(M) \rightarrow$ $H_{*}\left(B \mathbf{Z}_{p^{r}}\right)$ has $\Psi_{*}(G)=0$. Moreover, one can use $W$ to calculate $\sigma(M, \Psi)$ 
and it follows from Lemma 8 that $\sigma(M, \Psi)=0$. Proposition 2 calculates $\Psi_{*}=(s \psi)_{*}+\left(t \psi^{\prime}\right)_{*}$; evaluating on $x=\alpha f+f^{\prime}$ gives (ii) after simplification. Finally, $\sigma(M, \Psi)=\sigma(L, s \psi)-\sigma\left(L^{\prime}, t \psi^{\prime}\right)$ so $\sigma(L, \psi)=\sigma\left(L^{\prime}, t \psi^{\prime}\right)$.

The conclusion of Theorem 10 looks pretty messy but it simplifies somewhat for the class 9 of lens spaces which we know imbed punctured. For these, Theorem 10 yields:

COROLlaRY 11. Suppose $a$ and c satisfy $\left(*_{2 k}\right)$. If

$$
\begin{aligned}
& L\left(m ; 1, a, \ldots, a^{k-1}, b, b a, \ldots, b a^{k-1}\right) \# \\
& \quad-L\left(m ; 1, c, \ldots, c^{k-1}, d, d c, \ldots, d c^{k-1}\right)
\end{aligned}
$$

imbeds in $S^{4 k}$, then for a prime $p$ with $p^{r} \mid m$ but $p^{r+1} \nmid m$, we have: For all $s$, there exist numbers $\alpha$ and $t$ such that

(i) $\alpha^{2} b^{k} \equiv d^{k}\left(\bmod p^{r}\right)$

(ii) $s^{k}+\alpha t^{k} \equiv 0\left(\bmod p^{r}\right)$

(iii) $\sigma(L, s \psi)=\sigma\left(L^{\prime}, t \psi^{\prime}\right)$

Proof. Just write everything in terms of $a, b, c$ and $d$ : The condition on $s, t$, and $\alpha$ is $\alpha^{2} a^{k(2 k-1)} \cdot b^{k} \equiv c^{k(2 k-1)} d^{k}$, and

$$
0=\alpha s^{k} c^{k(k-1) / 2} b^{k} a^{k(k-1) / 2}+t^{k} a^{k(k-1) / 2} \cdot d^{k} c^{k(k-1) / 2} .
$$

Since $a$ and $c$ both satisfy $\left(*_{2 k}\right), 0=a^{2 k}-1=\left(a^{k}-1\right)\left(a^{k}+1\right)$ implies that $a^{k}=-1$ and likewise that $c^{k} \equiv-1$. Substituting this into the first equality gives (i). The second simplifies as well once we note that $a$ and $c$ are both units, and so the result follows.

To see how this works in a particular case, here is an example, calculated by computer.

EXAMPLE. Let $m=222=13 \cdot 17, d=13$ and $k=2$. Then

$$
L(221 ; 1,21,1,21) \#-L(221 ; 1,47,1,47)
$$

does not imbed in $S^{8}$ although each summand imbeds punctured.

Proof. $a=21$ and $c=47$ clearly satisfy the condition $\left(*_{4}\right)$, so both summand imbed punctured. The solutions of $z^{2} \equiv 1(\bmod 13)$ are $\alpha= \pm 1$, and the solutions of $z^{2} \equiv-1(\bmod 13)$ are $t= \pm 5$, so the corollary says (for $s=1$ ) that $\sigma(L, \psi)=\sigma\left(L^{\prime}, \pm \psi^{\prime}\right)$ or $\sigma\left(L^{\prime}, \pm 5 \psi^{\prime}\right)$. But by computer calculation, $\sigma(L, \psi)=212,245 / 221$ whereas $\sigma\left(L^{\prime}, \pm 5 \psi^{\prime}\right)=\sigma\left(L, \pm \psi^{\prime}\right)=$ $63,733 / 221$. Hence $M$ does not imbed in $S^{8}$. 
The original motivation for this investigation was its relation to double null-concordance of even-dimensional knots. There are two knots $K$ and $K^{\prime}$ arising from $\partial L_{0}$ and $\partial L_{0}^{\prime}$ as sitting in $S^{8}$. The methods of [11] can be used to show that $K \#-K^{\prime}$ is a knot which is algebraically but not geometrically doubly slice.

Note added in proof. The author and S. Cappell (to appear) have investigated the questions raised in this paper more fully for $\left|\pi_{1} L\right|$ divisible by more than one prime. We have necessary and sufficient conditions for punctured imbeddings and imbeddings of connected sums. Our results extend as well to non-linear lens spaces.

\section{REFERENCES}

[1] M. F. Atiyah and R. Bott, A Lefschetz fixed point formula for elliptic complexes: II. Applications, Ann. of Math., 88 (1968), 451-491.

[2] A. Casson and C. Gordon, Cobordism of classical knots, (preprint), Orsay 1975.

[3] M. Cohen, A Course in Simple-Homotopy Theory, Springer-Verlag, New York, 1973.

[4] P. Conner and E. Floyd, Differentiable Periodic Maps, Springer-Verlag, Berlin, 1964.

[5] D. Epstein, Embedding punctured manifolds, Proc. Amer. Math. Soc., 16 (1965), 175-176.

[6] J. Ewing, S. Moolgavkor, L. Smith and R. Stong, Stable parallelizability of lens spaces, J. Pure Appl. Algebra, 10 (1977), 177-192.

[7] P. Gilmer, Configurations of surfaces in 4-manifolds, Trans. Amer. Math. Soc., 264 (1981), 353-380.

[8] P. Gilmer and C. Livingston, On embedding 3-manifolds in 4-space, (to appear).

[9] J. Milnor, Lectures on the $h$-Cobordism Theorem, Princeton University Press, Princeton, 1965.

[10] G. deRham, Sur l'analysis situs des variétés à n dimensions, J. Math. Pures et Appl., 10 (1931), 115-200.

[11] D. Ruberman, Doubly slice knots and the Casson-Gordon invariants, Trans. Amer. Math. Soc., 279 (1983), 569-588.

Received September 6, 1982.

COURANT INSTITUTE

NEW YORK UNIVERSITY

NEW YORK, NY 10012 



\section{PACIFIC JOURNAL OF MATHEMATICS \\ EDITORS}

Donald BaBBITT (Managing Editor)

University of California

Los Angeles, CA 90024

Hugo RossI

University of Utah

Salt Lake City, UT 84112

C. C. Moore and Arthur Ogus

University of California

Berkeley, CA 94720
J. DugundiI

Department of Mathematics

University of Southern California

Los Angeles, CA 90089-1113

R. FINN and H. SAMELSON

Stanford University

Stanford, CA 94305

\section{ASSOCIATE EDITORS}
R. ARENS
E. F. BECKENBACH
B. H. NeUMANN
F. WOLF
K. YoshidA (1906-1982)

\section{SUPPORTING INSTITUTIONS}

\author{
UNIVERSITY OF ARIZONA \\ UNIVERSITY OF BRITISH COLUMBIA \\ CALIFORNIA INSTITUTE OF TECHNOLOGY \\ UNIVERSITY OF CALIFORNIA \\ MONTANA STATE UNIVERSITY \\ UNIVERSITY OF NEVADA, RENO \\ NEW MEXICO STATE UNIVERSITY \\ OREGON STATE UNIVERSITY
}

\author{
UNIVERSITY OF OREGON \\ UNIVERSITY OF SOUTHERN CALIFORNIA \\ STANFORD UNIVERSITY \\ UNIVERSITY OF HAWAII \\ UNIVERSITY OF TOKYO \\ UNIVERSITY OF UTAH \\ WASHINGTON STATE UNIVERSITY \\ UNIVERSITY OF WASHINGTON
}

The Supporting Institutions listed above contribute to the cost of publication of this Journal, but they are not owners or publishers and have no responsibility for its content or policies.

Mathematical papers intended for publication in the Pacific Journal of Mathematics should be in typed form or offset-reproduced (not dittoed), double spaced with large margins. Please do not use built up fractions in the text of the manuscript. However, you may use them in the displayed equations. Underline Greek letters in red, German in green, and script in blue. The first paragraph must be capable of being used separately as a synopsis of the entire paper. In particular it should contain no bibliographic references. Please propose a heading for the odd numbered pages of less than 35 characters. Manuscripts, in triplicate, may be sent to any one of the editors. Please classify according to the scheme of Math. Reviews, Index to Vol. 39. Supply name and address of author to whom proofs should be sent. All other communications should be addressed to the managing editor, or Elaine Barth, University of California, Los Angeles, California 90024.

There are page-charges associated with articles appearing in the Pacific Journal of Mathematics. These charges are expected to be paid by the author's University, Government Agency or Company. If the author or authors do not have access to such Institutional support these charges are waived. Single authors will receive 50 free reprints; joint authors will receive a total of 100 free reprints. Additional copies may be obtained at cost in multiples of 50 .

The Pacific Journal of Mathematics is issued monthly as of January 1966. Regular subscription rate: $\$ 132.00$ a year (6 Vol., 12 issues). Special rate: $\$ 66.00$ a year to individual members of supporting institutions.

Subscriptions, orders for numbers issued in the last three calendar years, and changes of address should be sent to Pacific Journal of Mathematics, P.O. Box 969, Carmel Valley, CA 93924, U.S.A. Old back numbers obtainable from Kraus Periodicals Co., Route 100, Millwood, NY 10546.

The Pacific Journal of Mathematics ISSN 0030-8730 is published monthly by the Pacific Journal of Mathematics at P.O. Box 969, Carmel Valley, CA 93924. Application to mail at Second-class postage rates is pending at Carmel Valley, California, and additional mailing offices. Postmaster: Send address changes to Pacific Journal of Mathematics, P. O. Box 969, Carmel Valley, CA 93924.

PUBLISHED BY PACIFIC JOURNAL OF MATHEMATICS, A NON-PROFIT CORPORATION

Copyright $\odot 1984$ by Pacific Journal of Mathematics 


\section{Pacific Journal of Mathematics}

\section{Vol. 113, No. $2 \quad$ April, 1984}

Alan Adolphson, On the Dwork trace formula ...................257

Amos Altshuler and Leon Steinberg, Enumeration of the quasisimplicial

3 -spheres and 4-polytopes with eight vertices .................. 269

Kenneth R. Goodearl, Cancellation of low-rank vector bundles .......... 289

Gary Fred Gruenhage, Ernest A. Michael and Yoshio Tanaka, Spaces

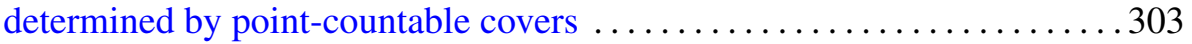

Charles Lemuel Hagopian, Atriodic homogeneous continua .......... 333

David Harbater, Ordinary and supersingular covers in characteristic $p$. . . 349

Domingo Antonio Herrero, Continuity of spectral functions and the lakes

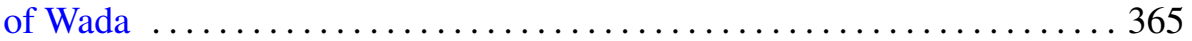

Donald William Kahn, Differentiable approximations to homotopy resolutions and framed cobordism ....................... 373

K. McGovern, On the lifting theory of finite groups of Lie type $\ldots \ldots \ldots . \ldots 383$

C. David (Carl) Minda, The modulus of a doubly connected region and the geodesic curvature-area method ............................. 395

Takuo Miwa, Complexes are spaces with a $\sigma$-almost locally finite base $\ldots . .407$

Ho Kuen Ng, Finitely presented dimension of commutative rings and modules

Roger David Nussbaum, A folk theorem in the spectral theory of

$C_{0}$-semigroups

J. S. Okon, Prime divisors, analytic spread and filtrations

Harold Raymond Parks, Regularity of solutions to elliptic isoperimetric problems

R. Sitaramachandra Rao and M. V. Subba Rao, Transformation formulae for multiple series

Daniel Ruberman, Imbedding punctured lens spaces and connected sums

Uri Srebro, Deficiencies of immersions 